\title{
Analysis of effects of macroscopic propagation and multiple molecular orbitals on the minimum in high-order harmonic generation of aligned $\mathrm{CO}_{2}$
}

\author{
Cheng Jin, ${ }^{1}$ Anh-Thu Le, ${ }^{1}$ and C. D. Lin $^{1}$ \\ ${ }^{1}$ J. R. Macdonald Laboratory, Physics Department, \\ Kansas State University, Manhattan, Kansas 66506-2604, USA
}

(Dated: May 11, 2021)

\begin{abstract}
We report theoretical calculations on the effect of the multiple orbital contribution in high-order harmonic generation (HHG) from aligned $\mathrm{CO}_{2}$ with inclusion of macroscopic propagation of harmonic fields in the medium. Our results show very good agreements with recent experiments for the dynamics of the minimum in HHG spectra as laser intensity or alignment angle changes. Calculations are carried out to check how the position of the minimum in HHG spectra depends on the degrees of molecular alignment, laser focusing conditions, and the effects of alignment-dependent ionization rates of the different molecular orbitals. These analyses help to explain why the minima observed in different experiments may vary.
\end{abstract}

PACS numbers: $33.80 . \mathrm{Rv}, 42.65 . \mathrm{Ky}, 31.70 . \mathrm{Hq}, 33.80 . \mathrm{Eh}$

\section{INTRODUCTION}

High-order harmonic generation (HHG) is one of the most interesting nonlinear phenomena when atoms or molecules are exposed to an intense laser field [1-3]. Experimentally the harmonic fields generated by all atoms or molecules within the laser focus co-propagate with the fundamental laser field till they reach the detector. To compare with experimental measurements, theoretical harmonics from each atom or molecule first have to be calculated. The induced dipoles are then fed into the propagation equation, taking into account the focusing condition, the nature of the gas medium, and the equation is finally integrated till the harmonics are collected [4 8).

HHG from randomly distributed molecules have been studied since the 1990's [9 12]. In recent years, experimental HHG studies tend to focus on partially aligned molecules [13 16]. Among the molecules, $\mathrm{CO}_{2}$ is likely the most extensively studied system so far [17 20]. Initially the interest was focused on the observation of the minimum in the HHG spectra of $\mathrm{CO}_{2}$ [21 23]. The positions of the minima from different experiments, however, are often vastly different. According to the three-step model [24, 25], HHG is generated through the recombination of the returning electrons with the molecular ion. The interference of electron waves from the two atomic centers, under some conditions, results in a minimum in the transition dipole, the simplest is the two-center interference model [26 28]. In fact, recombination is an inverse process of photoionization. Thus the transition dipole is the same as that in photoionization. Any such minima have not been observed in photoionization, however, since in these measurements molecules are isotropically distributed.

From the theoretical side, the alignment dependence of HHG was first studied using the strong-field approximation (SFA) (or the Lewenstein model) 29 31]. Subsequently we developed a quantitative rescattering (QRS) theory [32 34] for HHG which was a significant improvement on the Lewenstein model. In QRS the accuracy of recombination is treated at the same level as in the photoionization process. Using QRS, the HHG minimum is attributed to the minimum in the photoionization transition dipole between the highest occupied molecular orbital (HOMO) and continuum molecular wave functions 34, 35]. For fixed-in-space $\mathrm{CO}_{2}$ molecules the photoionization cross sections of HOMO indeed exhibit minima at small alignment angles. The experimental HHG spectra from partially aligned $\mathrm{CO}_{2}$ have been explained reasonably well by QRS, including the harmonic intensities and the phase, so are the polarization and ellipticity of the harmonics [35, 36].

If HHG is generated from HOMO only, then one expects that the position of the minimum does not significantly change with laser intensity according to QRS theory. Indeed, strong field ionization depends exponentially on the ionization potential $\mathrm{I}_{\mathrm{p}}$. The HOMO-1 and HOMO-2 orbitals in $\mathrm{CO}_{2}$ are 4 and $4.4 \mathrm{eV}$ more deeply bound than the HOMO [37], thus they are not expected to contribute significantly to the HHG spectra. However, it is also well known that tunneling ionization rates depend sensitively on the symmetry of the molecular orbital [38]. The HOMO is a $\pi_{g}$ orbital. It means that at small alignment angles the ionization rates are small. For HOMO-2, on the other hand, it is a $\sigma_{g}$ orbital, thus it has large ionization rate when $\mathrm{CO}_{2}$ molecules are parallel aligned. Thus for small alignment angles, HOMO-2 may become important even though it is bound $4.4 \mathrm{eV}$ deeper than the HOMO. (HOMO-1 is a $\pi_{u}$ orbital and thus not expected to contribute significantly to the HHG.)

The first step of HHG process is tunneling ionization. The alignment dependence of tunneling ionization rate is usually calculated using molecular Ammosov-DeloneKrainov (MO-ADK) theory [39, 40] or SFA. For most molecules that have been studied the two models give nearly identical alignment dependence (after normalization). However, this is not the case for $\mathrm{CO}_{2}$. Experimentally, the alignment dependence of $\mathrm{CO}_{2}$ ionization 
reported by Pavičić et al. [41] is very narrowly peaked near alignment angle of $46^{\circ}$. It differs significantly from the predictions of MO-ADK and SFA [42]. In fact, so far all theoretical attempts [43 47] have not been able to confirm the sharp alignment dependence reported in the experiment. Furthermore, the observed HHG spectra from aligned molecules are inconsistent with the reported experimental alignment dependence of ionization [36, 48].

The HHG spectra of parallel aligned $\mathrm{CO}_{2}$ have been studied in a number of experiments, with 800 -nm lasers [21] as well as lasers of longer wavelengths [23, 49]. It was observed that the position of the HHG minimum moved to higher photon energies as the laser intensity was increased. In Smirnova et al. [21], such effects were interpreted in terms of the multi-channel interference (between HOMO and HOMO-2) and the intricate hole dynamics. Their calculations were based on HHG generated by a single-molecule response. They introduced filters into the theory such that only short trajectories contributed to the signals. In their calculation, a relative phase between different channels due to strong-field ionization step was introduced "by hand" in order to obtain the good agreement with the measurement.

As noted at the beginning, to compare theoretically simulated HHG spectra of molecules with experimental ones, the effect of macroscopic propagation should be considered. Practically, this has rarely been done. For atomic targets, propagation effect is usually included with single-atom induced dipoles calculated using the Lewenstein model. Only in a few rare occasions the atomic response is calculated more accurately by solving the time-dependent Schrödinger equation (TDSE) [50, 51]. It is also well-known that the Lewenstein model does not predict the HHG spectra (the intensity) precisely, but the phases of the harmonics are relatively accurate. This fact is used in the QRS model, which can be understood as simply replacing the transition dipole calculated using plane waves in the Lewenstein model by one calculated using accurate molecular continuum wave functions. The improvement of single-molecule HHG spectra calculated using QRS has been well documented in our previous publications [34, 52]. Computationally, QRS is nearly as simple as the Lewenstein model (actually QRS is even simpler than SFA for polyatomic molecules, see Ref. [53]), thus induced dipole can be easily obtained from QRS to feed into the propagation equations to account for medium propagation effects. This has been done for atomic targets for lowlaser-intensity and low-gas-pressure conditions [54]. It has been extended recently to the conditions of high intensity and high pressure for Ar and to molecular targets [8, 55], including polyatomic molecules [53].

In this paper, we report theoretical studies of the propagation effect on the $\mathrm{HHG}$ of $\mathrm{CO}_{2}$, including contributions from HOMO and HOMO-2. Note that experimentally the effect of propagation on the HHG spectra has rarely been investigated either, in particular, its effect on the minimum of the HHG spectra. However, this has changed recently with the reports of such studies on $\mathrm{Ar}$ [55 57]. The rest of this paper is arranged as follows. In Sec. II, we first summarize the equations used for the propagation calculations. We limit ourselves to low laser intensity and low pressure. To extend the theory to high intensity and high pressure, the optical properties of $\mathrm{CO}_{2}$ have to be used and they are not available over a broad range of frequencies. We then summarize how the single-molecule response from partially aligned molecules is calculated. In Sec. III the calculated results are presented. Different factors that can influence the precise positions of the HHG minima are examined and reported. These results show that precise theoretical predictions of the positions of HHG minima in a given experiment is difficult, but the trend (the direction of the shift of the minimum positions) can be predicted (or explained). A short summary at the end concludes this paper.

\section{THEORETICAL METHOD}

We first briefly describe the theory of propagation of high harmonics in a macroscopic medium, and in free space, till they reach the detector. To calculate the induced dipole for individual molecules, we include the contributions from multiple molecular orbitals. Our method is based on extending the QRS theory [34].

\section{A. Propagation of harmonics in the medium}

In general, both the fundamental laser field and the harmonic field are modified when they co-propagate through a macroscopic medium. In this paper, we limit ourselves to experiments carried out under the conditions of low laser intensity and low gas pressure, where the effects of dispersion, Kerr nonlinearity, and plasma defocusing on the fundamental laser field can be neglected [8, 54]. The fundamental laser field is assumed to be a Gaussian beam in space. Its spatial and temporal dependence can be expressed in an analytical form [54]. For high harmonics, dispersion and absorption effects from the medium are not included. The dispersion and absorption coefficients depend linearly on gas pressure and could be ignored under low pressure [8]. The free-electron dispersion is also neglected since the plasma frequency is much smaller than the frequencies of high harmonics [5].

The three-dimensional propagation equation of the harmonic field is described as [4 [6, 8, 54]

$$
\begin{aligned}
\nabla_{\perp}^{2} \tilde{E}_{\mathrm{h}}^{\|}\left(r, z^{\prime}, \omega, \alpha\right)- & \frac{2 i \omega}{c} \frac{\partial \tilde{E}_{\mathrm{h}}^{\|}\left(r, z^{\prime}, \omega, \alpha\right)}{\partial z^{\prime}} \\
& =-\omega^{2} \mu_{0} \tilde{P}_{\mathrm{nl}}^{\|}\left(r, z^{\prime}, \omega, \alpha\right),
\end{aligned}
$$

where

$$
\tilde{E}_{\mathrm{h}}^{\|}\left(r, z^{\prime}, \omega, \alpha\right)=\hat{F}\left[E_{\mathrm{h}}^{\|}\left(r, z^{\prime}, t^{\prime}, \alpha\right)\right]
$$


and

$$
\tilde{P}_{\mathrm{nl}}^{\|}\left(r, z^{\prime}, \omega, \alpha\right)=\hat{F}\left[P_{\mathrm{nl}}^{\|}\left(r, z^{\prime}, t^{\prime}, \alpha\right)\right] .
$$

Here $\hat{F}$ is the Fourier transform operator acting on the temporal coordinate. Eq. (1) is written in a moving coordinate frame $\left(z^{\prime}=z\right.$ and $\left.t^{\prime}=t-z / c\right)$ and the term $\partial^{2} E_{\mathrm{h}}^{\|} / \partial z^{\prime 2}$ is neglected. $\tilde{E}_{\mathrm{h}}^{\|}\left(r, z^{\prime}, \omega, \alpha\right)$ is the parallel component of the harmonic field with respect to the polarization direction of the probe (or generating) laser, and $\tilde{P}_{\mathrm{nl}}^{\|}\left(r, z^{\prime}, \omega, \alpha\right)$ is the parallel component of the nonlinear polarization caused by the generating laser field, where $\alpha$ is pump-probe angle, i.e., the angle between the aligning laser and the harmonic generating laser polarizations.

The nonlinear polarization term can be expressed as

$\tilde{P}_{\mathrm{nl}}^{\|}\left(r, z^{\prime}, \omega, \alpha\right)=\hat{F}\left\{\left[n_{0}-n_{\mathrm{e}}\left(r, z^{\prime}, t^{\prime}, \alpha\right)\right] D^{\|, \text {tot }}\left(r, z^{\prime}, t^{\prime}, \alpha\right)\right\}$,

where $n_{0}$ is the density of neutral molecules, $D^{\| \text {,tot }}\left(t^{\prime}, \alpha\right)$ is the parallel component of the induced single-molecule dipole over a number of active electrons [see Eq. (12) below], and $n_{\mathrm{e}}\left(t^{\prime}, \alpha\right)$ is the free-electron density, which can be calculated as following:

$$
n_{\mathrm{e}}\left(t^{\prime}, \alpha\right)=\int_{0}^{\pi} n_{\mathrm{e}}\left(t^{\prime}, \theta\right) \rho(\theta, \alpha) \sin \theta d \theta .
$$

Here $n_{\mathrm{e}}\left(t^{\prime}, \theta\right)$ is the alignment-dependent free-electron density, obtained from

$$
n_{\mathrm{e}}\left(t^{\prime}, \theta\right)=n_{0}\left\{1-\exp \left[-\int_{-\infty}^{t^{\prime}} \gamma(\tau, \theta) d \tau\right]\right\},
$$

where $\gamma(\tau, \theta)$ is the alignment-dependent ionization rate, which can be calculated by MO-ADK theory [39, 40] for different molecular orbitals. In Eq. (5), $\theta$ is the alignment angle with respect to the polarization direction of the probe laser, and $\rho(\theta, \alpha)$ is the alignment distribution with pump-probe angle $\alpha$ [34, 58, 59].

After the propagation in the medium, we obtain the parallel component of near-field harmonics on the exit face of the gas jet $\left(z^{\prime}=z_{\text {out }}\right)$. For isotropically distributed molecules and partially aligned molecules with $\alpha=0^{\circ}$ or $90^{\circ}$, by symmetry there are only parallel harmonic components. The perpendicular components, which are usually much smaller, would appear for partially aligned molecules and the harmonics will be elliptically polarized in general [36]. Generalization of Eq. (1) to the perpendicular component is straightforward, but we restrict ourselves to parallel component only. Eq. (1) is solved numerically using a Crank-Nicholson routine for each value of $\omega$. Typical parameters used in the calculations are 300 grid points along the radial direction and 400 grid points along the longitudinal direction.

\section{B. Harmonics in the far field}

Once the near-field harmonics are obtained on the exit face of the medium, they further propagate in free space before detected by the spectrometer. In the meanwhile, they may pass through a slit, an iris, or be reflected by a mirror. The far-field harmonics can be obtained from near-field harmonics through a Hankel transformation 60 62.

$$
\begin{aligned}
E_{\mathrm{h}}^{\mathrm{f}}\left(r_{\mathrm{f}}, z_{\mathrm{f}}, \omega, \alpha\right)= & -i k \int \frac{\tilde{E}_{\mathrm{h}}^{\|}\left(r, z^{\prime}, \omega, \alpha\right)}{z_{\mathrm{f}}-z^{\prime}} J_{0}\left(\frac{k r r_{\mathrm{f}}}{z_{\mathrm{f}}-z^{\prime}}\right) \\
& \times \exp \left[\frac{i k\left(r^{2}+r_{\mathrm{f}}^{2}\right)}{2\left(z_{\mathrm{f}}-z^{\prime}\right)}\right] r d r
\end{aligned}
$$

where $J_{0}$ is the zero-order Bessel function, $z_{\mathrm{f}}$ and $r_{\mathrm{f}}$ are the coordinates of the far-field points. The wave vector $k$ is given by $k=\omega / c$.

We assume that the harmonics in the far field are col(4) lected from an extended area. By integrating harmonic yields over the area, the power spectrum of the macroscopic harmonics is obtained by

$$
S_{\mathrm{h}}(\omega, \alpha) \propto \iint\left|E_{\mathrm{h}}^{\mathrm{f}}\left(x_{\mathrm{f}}, y_{\mathrm{f}}, z_{\mathrm{f}}, \omega, \alpha\right)\right|^{2} d x_{\mathrm{f}} d y_{\mathrm{f}},
$$

where $x_{\mathrm{f}}$ and $y_{\mathrm{f}}$ are the Cartesian coordinates on the plane perpendicular to the propagation direction, and $r_{\mathrm{f}}=\sqrt{x_{\mathrm{f}}^{2}+y_{\mathrm{f}}^{2}}$. To simulate experimental HHG spectra quantitatively, besides laser parameters, detailed information on the experimental setup is needed.

\section{Quantitative rescattering theory for a multi-orbital molecular system}

In Eq. (4), laser induced single-molecule dipole moment $D\left(t^{\prime}\right)$ is calculated quantum mechanically using the QRS theory, which has been discussed in detail for molecules in Ref. 34]. Within QRS, laser induced dipole moment $D(\omega, \theta)$ for a molecule at a fixed angle $\theta$ (measured with respect to laser polarization) is given by

$$
D^{\|, \perp}(\omega, \theta)=N(\theta)^{1 / 2} W(\omega) d^{\|, \perp}(\omega, \theta),
$$

where $N(\theta)$ is the alignment-dependent ionization probability, $W(\omega)$ is the recombining electron wave packet, and $d^{\|, \perp}(\omega, \theta)$ is the parallel component (or perpendicular component) of the photorecombination (PR) transition dipole (complex in general). Here we only consider linearly polarized lasers and linear molecules. $W(\omega)$ is independent of the alignment angle $\theta$. From Eq. (9), it can be expressed as

$$
W(\omega)=\frac{D^{\|, \perp}(\omega, \theta)}{N(\theta)^{1 / 2} d^{\|, \perp}(\omega, \theta)} .
$$

In QRS, $W(\omega)$ is usually calculated only once for a given angle $\theta$ using SFA, where all the elements on the right-hand side of Eq. (10) are obtained by replacing the continuum waves with plane waves. In QRS, accurate $d^{\|, \perp}(\omega, \theta)$ are obtained from quantum chemistry code 
[63, 64] and tunneling ionization probability $N(\theta)$ are obtained either from MO-ADK [39, 40] or from SFA. Put all of these together into Eq. (9), laser induced dipole moment $D(\omega, \theta)$ for each orbital is obtained. Note that in QRS, the wave packet $W(\omega)$ automatically includes the phase which is dependent on the molecular orbital. Thus there is no need to introduce the relative phase between orbitals, in contrast to the approach in Ref. [21]. SFA is used to calculate the ionization probability in Eq. (9) in this paper unless otherwise stated.

We assume that the degree of molecular alignment is not varied spatially within the medium because the molecules are usually partially aligned by a weak and loosely focused laser beam [58]. By coherently averaging the induced dipole moments over the molecular angular distribution, we obtain the averaged induced dipole of partially aligned molecules at each point inside the medium:

$$
D^{\|, \operatorname{avg}}(\omega, \alpha)=\int_{0}^{\pi} D^{\|}(\omega, \theta) \rho(\theta, \alpha) \sin \theta d \theta,
$$

where $\rho(\theta, \alpha)$ is again the angular (or alignment) distribution of the molecules with respect to the polarization direction of the probe laser. For randomly distributed molecules, $\rho(\theta, \alpha)$ is a constant. Note that the above procedure is only for the specified molecular orbital.

For the specific problem addressed in this paper, we consider electrons either in the HOMO $\left(1 \pi_{g}\right)$ or in the HOMO-2 $\left(3 \sigma_{u}\right)$ of $\mathrm{CO}_{2}$. The electrons are freed and then recombine back to the same orbital. As discussed above, the multiple orbital effects are important at small alignment angles only due to symmetry consideration. At these angles HOMO-1 $\left(1 \pi_{u}\right)$ is ignored since the ionization rate of HOMO-1 is quite small compared with HOMO and HOMO-2 21, 40, 43. At large alignment angles, only HOMO becomes important. The total laser induced dipole over a number of active electrons can be written as 65,66 .

$$
D^{\|, \operatorname{tot}}(\omega, \alpha)=\sum_{j, n} D_{j, n}^{\|, \operatorname{avg}}(\omega, \alpha),
$$

where index $j$ refers to the $\mathrm{HOMO}$ and $\mathrm{HOMO}-2$ of $\mathrm{CO}_{2}$, and $n$ is an index to account for degeneracy in each molecular orbital.

Before proceeding, we comment that the QRS theory is formulated in the energy (or frequency) domain. There is no explicit treatment of "core dynamics" as in Ref. [21]. The time evolution of the core is reflected only by the energy of the core in the free propagation of the electron wave packet. In other words, any possible many-body interchannel couplings between HOMO and HOMO-2 are not included in the present treatment. Before such effects are addressed, other factors that are more important on the HHG as discussed here have to be treated first.

For the propagation of harmonics in the medium, we need to obtain hundreds of the total induced dipoles $D^{\| \text {,tot }}(\omega, \alpha)$ for different laser intensities, and then they are fed into Eq. (11). The same procedure is used in Jin et al. 54 for atomic targets. Note that in the present model, dielectric properties of molecules due to non-isotropic distributions are also neglected.

\section{RESULTS AND DISCUSSION}

\section{A. HHG spectra of randomly distributed $\mathrm{CO}_{2}$ : theory vs experiment}

HHG spectra by $800-\mathrm{nm}$ and $1200-\mathrm{nm}$ lasers have been reported for isotropically distributed and partially aligned $\mathrm{N}_{2}$ and $\mathrm{CO}_{2}$ molecules 49]. The spectra of $\mathrm{N}_{2}$ have been analyzed by Jin et al. 8, 55] recently including only the HOMO.

In Figs. 11(a)-11(c), we show the HHG spectra for isotropically distributed $\mathrm{CO}_{2}$ molecules by $800-\mathrm{nm}$ and 1200-nm lasers. To obtain good agreement between theory and experiment, especially in the cutoff region, the intensity used in the theory is adjusted from the value given in the experiment. In Figs. 1(a)-1(c), the intensities in theory (experiment) are $1.9(2.1), 0.8(1.0), 1.0$ (1.2), in units of $10^{14} \mathrm{~W} / \mathrm{cm}^{2}$, respectively. Other parameters used in the simulation are the same as those given in the experiment 49]. The laser parameters are: pulse duration is $\sim 32 \mathrm{fs}(800 \mathrm{~nm})$ or $\sim 44 \mathrm{fs}(1200 \mathrm{~nm})$, beam waist at the focus is $\sim 40 \mu \mathrm{m}$. A $0.6-\mathrm{mm}$-wide gas jet is located $3 \mathrm{~mm}(800 \mathrm{~nm})$ or $3.5 \mathrm{~mm}(1200 \mathrm{~nm})$ after the laser focus, and a slit with a width of $100 \mu \mathrm{m}$ is placed at $24 \mathrm{~cm}$ after the gas jet.

Figs. 1(a)-1(c) clearly show the good overall agreement between experiment and theory for randomly distributed $\mathrm{CO}_{2}$ molecules. We have checked that $\mathrm{HOMO}$ is dominant for randomly distributed $\mathrm{CO}_{2}$, with negligible contributions from inner orbitals. The HHG spectra do not exhibit any minima, as opposed to the spectra of random $\mathrm{N}_{2}$ molecules when they are generated under the same experimental conditions [49]. For randomly distributed $\mathrm{CO}_{2}$, there was no minimum found in HHG spectra using an 800-nm laser by Vozzi et al. [22]. However, for the 1300-nm lasers, the data from Torres et al. [23, 67] appear to show a very weak minimum at photon energy near $45 \mathrm{eV}$. Without more careful study including different intensities and wavelengths, however, this is not conclusive.

\section{B. HHG spectra of aligned $\mathrm{CO}_{2}$ : theory vs experiment}

Experimentally HHG spectra have also be reported from aligned $\mathrm{CO}_{2}$ molecules. A relatively weak and short laser pulse was used to impulsively align molecules, and the HHG spectra were taken at half-revival $(\sim 21.2$ ps in $\mathrm{CO}_{2}$ ) when the molecules were maximally aligned [49]. The angular distributions of the aligned molecules are obtained by solving the TDSE of rotational wave 


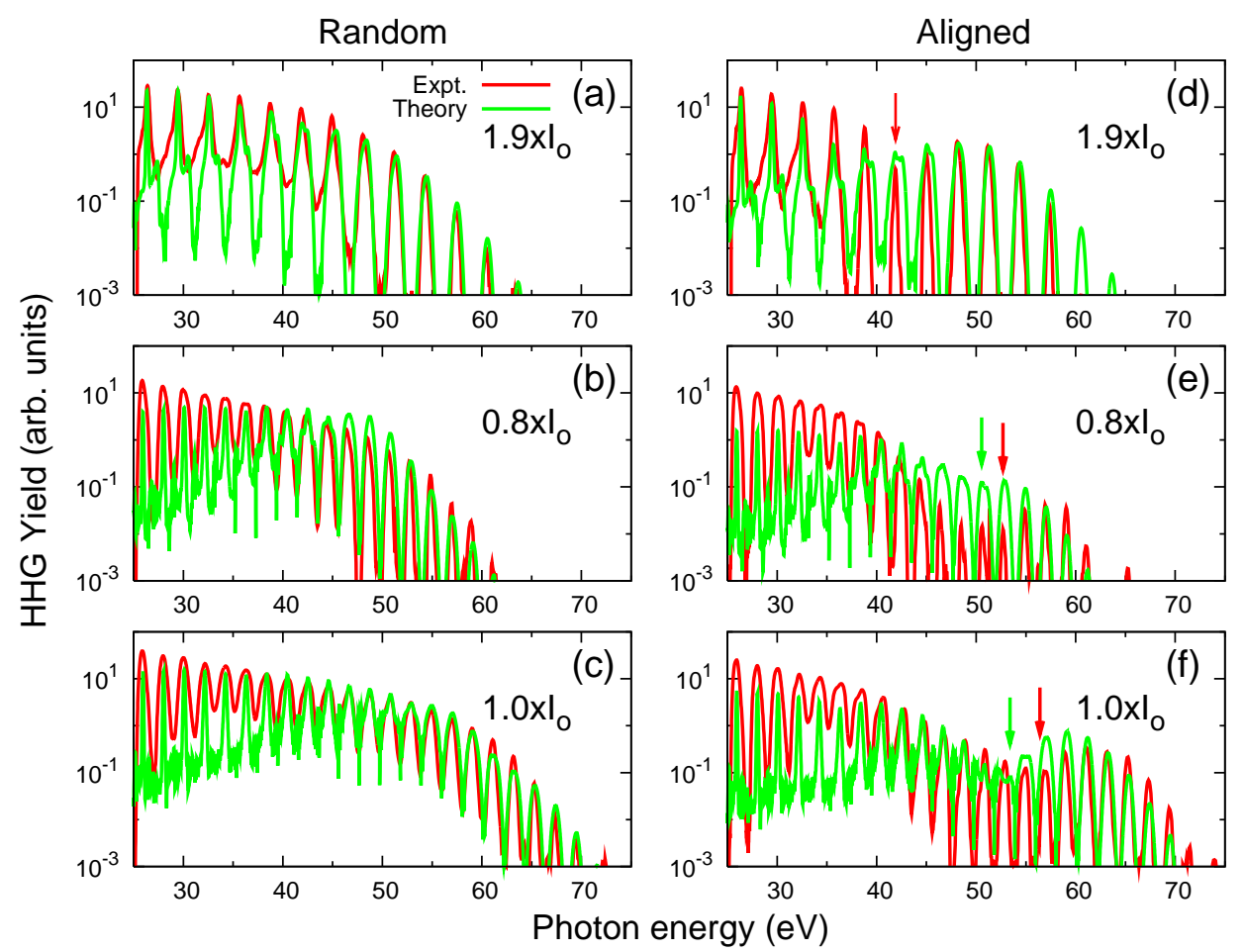

FIG. 1: (Color online) Comparison of theoretical (green lines) and experimental (red lines) HHG spectra of random and aligned $\mathrm{CO}_{2}$ molecules, in an 800-nm laser shown in (a) and (d), and in a 1200-nm laser shown in (b), (c), (e), and (f). Laser intensities are indicated where $\mathrm{I}_{\circ}=10^{14} \mathrm{~W} / \mathrm{cm}^{2}$. Experimental data are from Ref. [49]. Arrows indicate the positions of minima. Pump-probe angle $\alpha=0^{\circ}$. See text for additional laser parameters and experimental arrangements.

packet [58]. The degree of alignment is $\left\langle\cos ^{2} \theta\right\rangle=0.60$ in Fig. 1(d), and $\left\langle\cos ^{2} \theta\right\rangle=0.50$ in Figs. 1(e) and 1(f). The polarizations of the pump and probe lasers are parallel to each other.

The HHG spectra of partially aligned $\mathrm{CO}_{2}$ molecules are shown in Figs. 1(d)-1(f), which are obtained under the same generating lasers and experimental arrangements as those in Figs.11(a)-1(c), respectively. The simulation and experimental data agree well with each other in general. In Fig. 1(e), the discrepancy is a little bigger, showing the drop near $40 \mathrm{eV}$ is larger in the experiment than in the theory. But we note that in Fig. 1(f), the experimental data do not drop as rapidly, in agreement with the theoretical simulation.

The minima in the HHG spectra of $\mathrm{CO}_{2}$ and their dependence on laser intensity have been widely discussed in the literature [21, 23]. In Fig. 1(d), for an 800-nm laser, experiment gives a strong minimum at $42 \pm 2 \mathrm{eV}$, our simulation predicts a minimum around $42 \mathrm{eV}$. For the 1200-nm laser, in Fig. 1(e), experiment shows a minimum at $51 \pm 2 \mathrm{eV}$, theory predicts a minimum around $50 \mathrm{eV}$. In Fig. 1(f), the experimental minimum is shifted to $57 \pm 2 \mathrm{eV}$, and the theoretical one is moved to around $53.5 \mathrm{eV}$. Thus our simulation also shows the shift of the minimum from low to high harmonic orders as laser intensity is increased. Below we interpret the origin of the shift.

\section{Origin of minimum in the HHG spectra of aligned $\mathrm{CO}_{2}$ : Type I and Type II}

We next analyze the origin of the minimum in the HHG spectra seen in Figs.1(d)-1(f), and consider the dominant contributions from HOMO and HOMO-2 only. First we define the averaged PR transition dipole for each molecular orbital by

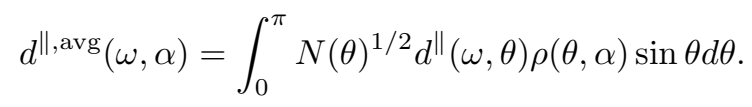

This gives a measure of the relative contribution of each molecular orbital to the HHG, which is obtained by averaging over the angular (or alignment) distribution of the partially aligned molecules, weighted by the square root of the tunneling ionization probability. The relative ionization rates between HOMO and HOMO-2 change with laser intensity.

Figs. 2(a)-2(c) show the envelopes of the HHG spectra from individual molecular orbitals together with the total ones, each obtained after propagation in the medium. In the meanwhile, the averaged PR transition dipoles of HOMO and HOMO-2 under different generating lasers and alignment distributions are shown in Figs. 2(d)-2(f), respectively.

In Figs. 2(a) and 2(b), there are no minima in the HHG spectra of HOMO or HOMO-2, but the minimum shows up in the total spectra. This is due to the interference between HOMO and HOMO-2. We call this 

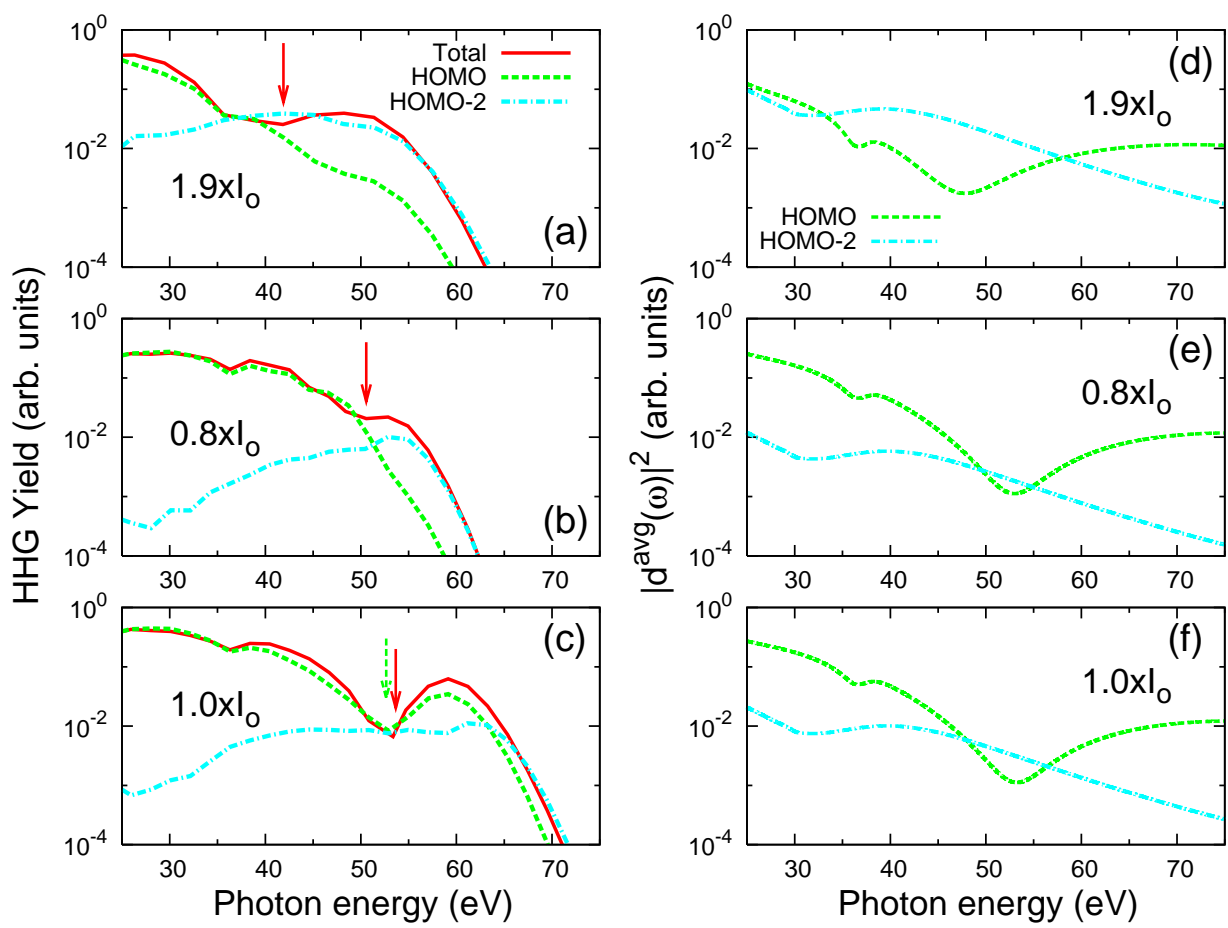

FIG. 2: (Color online) (a)-(c) Macroscopic HHG spectra (envelope only) corresponding to Figs. 11(d)-1(f), respectively. Total (HOMO and HOMO-2 together) spectra and the spectra of individual HOMO and HOMO-2 are shown. (d)-(f) Averaged photorecombination transition dipoles (parallel component, the square of magnitude) of HOMO and HOMO-2 corresponding to (a), (b), and (c), respectively. Laser intensities are indicated where $\mathrm{I}_{\circ}=10^{14} \mathrm{~W} / \mathrm{cm}^{2}$. Arrows indicate the positions of minima. Pump-probe angle $\alpha=0^{\circ}$.

type I minimum. Clearly the minimum position will change with laser intensity since the relative ionization rates between HOMO and HOMO-2 change with intensity [also see Figs. 5(c) and 5(d)]. Similar analysis can be found in Ref. [21]. In Fig. 2(c), there is a minimum in the HOMO spectra at $52.6 \mathrm{eV}$. This minimum is shifted to $53.6 \mathrm{eV}$ in the total spectra due to the interference with the HOMO-2. This is categorized as type II minimum. Similar analysis of this type can be found in Refs. [23, 68]. The minimum in the HOMO spectra is due to the minimum in the averaged PR transition dipole of HOMO shown in Fig. 2(f). But their positions differ due to modification of the macroscopic wave packet (MWP). In this connection we mention that the earlier calculations [34, 35] with an 800-nm laser showed minimum in HHG spectra at small pump-probe angles due to the contribution from the HOMO only. These calculations were carried out with a higher degree of alignment and higher laser intensities as compared to our present study. This is expected as the minimum in the averaged PR transition dipole from HOMO in Fig. 2(d) becomes deeper and is slightly shifted away from the cutoff to lower energies with increased degree of alignment [see Fig. 2(e) and [2(f)]. Furthermore, as shown in Ref. [55], the minimum in the HOMO spectra could also result from the multiplication of MWP and averaged PR transition dipole even when neither has minimum. When a minimum occurs in the dominant orbital, its position will not change much if it remains the dominant one when the laser intensity changes. The little bump around $36 \mathrm{eV}$ in the HOMO spectra as well as in the total spectra can be seen due to the bump in the HOMO curves in Figs. 2(d)-2(f). Its position does not change much since the HOMO-2 remains small.

Previously in Refs. [8, 55], we have shown that the macroscopic HHG spectrum is the product of a MWP and an averaged PR transition dipole for each individual molecular orbital. Since the ionization rate for each orbital has been incorporated in the averaged PR transition dipole, the MWP is mostly identical except for the phase due to ionization potential. The averaged PR transition dipole is very sensitive to ionization rates. The relative magnitude changes rapidly with the increase of laser intensity. Thus when two averaged PR transition dipoles are comparable [see Fig. 2(d)], the position of the minimum changes rapidly with the laser intensity. The averaged PR transition dipole is also sensitive to alignment distributions [see Figs. 2(d)-2(f)]. At low laser intensity, HOMO-2 is small, interference often occurs in a narrow region only where the two amplitudes are comparable, see Figs. 2(b) and 2(c). In comparison, in Smirnova et al. 21], HOMO and HOMO-2 tend to interfere over a broad photon-energy region. The ionization rates and transition dipoles used in their calculations are different from ours. 


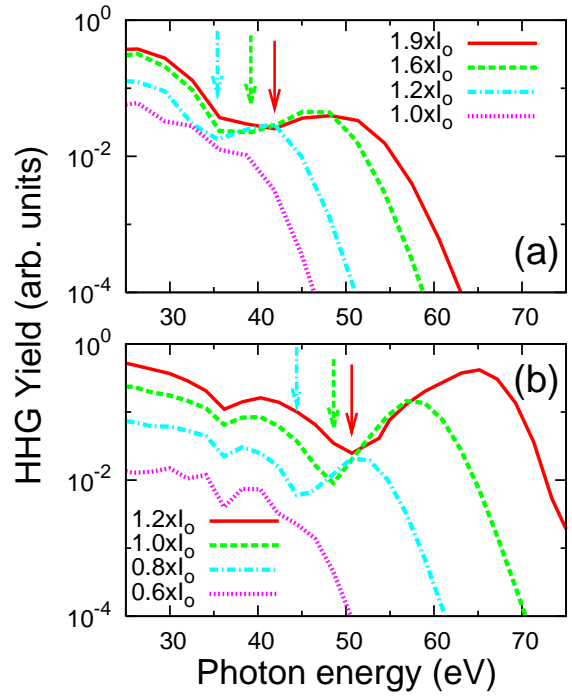

FIG. 3: (Color online) Laser intensity dependence of macroscopic HHG spectra (envelope only) (a) in an 800-nm laser and (b) in a 1200-nm laser. Intensities are shown in units of $\mathrm{I}_{\circ}=10^{14} \mathrm{~W} / \mathrm{cm}^{2}$. Arrows indicate the positions of minima. Degree of alignment: $\left\langle\cos ^{2} \theta\right\rangle=0.60$. Pump-probe angle $\alpha=0^{\circ}$.

\section{Progression of harmonic minimum vs laser intensity}

In Figs. 3(a) and 3(b) we show the envelope of the calculated HHG spectra for four different peak intensities with an 800-nm laser and a 1200-nm laser, respectively. For the $800-\mathrm{nm}$ spectra, the lowest intensity does not have a minimum. For the higher ones, each spectrum has a type I minimum, with its position shifts to lower photon energy as the laser intensity is decreased. The degree of alignment of molecules used in the calculation is $\left\langle\cos ^{2} \theta\right\rangle=0.60$. We find that the shift cannot be attributed to either MWP or the averaged PR transition dipole alone. For the 1200-nm data, also with $\left\langle\cos ^{2} \theta\right\rangle=0.60$, which is different from Figs. 1(e) and 1(f), we find that the minimum is type II, where the averaged $\mathrm{PR}$ transition dipole of the HOMO has a minimum. The minimum in the HHG spectra of the HOMO shifts to higher photon energy as the intensity increases, but the interference with HOMO-2 shifts the minimum to even higher energies. In other words, the shift of the position of the HHG minimum vs intensity cannot be attributed to a single factor alone.

\section{E. Other factors that influence the precise positions of HHG minima}

In our analysis, the averaged $\mathrm{PR}$ transition dipole is calculated over the angular distribution of the molecules and thus depend on the degrees of alignment. Since the latter cannot be accurately measured, we check how sensitive the calculated spectra is with respect to the as- sumed alignment distribution. In Fig. 4(c), four different alignment distributions are shown. The distributions are multiplied by the volume element $\sin \theta$ for easy comparison. Three of them are obtained from the calculated rotational wave packets [58], with $\left\langle\cos ^{2} \theta\right\rangle$ as $0.63,0.60$, and 0.55 , respectively. The other is the commonly used $\cos ^{4} \theta$ distribution. For $800-\mathrm{nm}$ and $1200-\mathrm{nm}$ lasers, the envelopes of the calculated HHG spectra are shown in Figs. 4(a) and 4(b), respectively. The precise position of the minimum changes slightly except for the one from the $\cos ^{4} \theta$ distributions. However, change of a couple of $\mathrm{eV}$ 's is seen.

To precisely determine the minimum in the HHG spectra, accurate alignment-dependent ionization probability $N(\theta)$ for each molecular orbital is needed. For $\mathrm{CO}_{2}$, even for HOMO, different theories in the literature 21, 4043, 45 47] show non-negligible differences, and they do not agree with the experimental data [41]. Here we examine how the HHG spectra change with the different ionization rates used. The ionization rates for both HOMO and HOMO-2 can all be easily calculated from SFA or from MO-ADK theory. Figs. 5(a) and 5(b) show the HHG spectra calculated using the ionization probabilities shown in Figs. 5(c) and5(d). Other laser parameters used in the calculation are given in the figure captions. The difference of the position of the minimum is $3 \mathrm{eV}$ in Fig. 5(a) and $2 \mathrm{eV}$ in Fig. 5(b). Note that the ionization probabilities from SFA and MO-ADK are normalized at the peak of the HOMO curve. In Fig. 5 (a) the spectra are normalized at H33 (51 eV) and in Fig. 5 (b) at H65 $(67 \mathrm{eV})$.

The HHG spectra are also sensitive to the experimental arrangement and thus can also move the position of the HHG minimum. To demonstrate this, we (i) move the gas jet to the laser focus and collect the signal using a slit; (ii) put the gas jet after the laser focus, and collect HHG signal without slit (total signal). These two will be compared to the arrangement used in this paper: gas jet is after the laser focus and the HHG is collected with a slit. The results are compared in Fig. 6, Note that the spectra are normalized at H17 (26 eV) in Fig. 6(a) and at H35 (36 eV) in Fig. 6(b). Not only the spectra change quite significantly, but also the position of the HHG minimum. This illustrates that it is very difficult to compare the position of the HHG minimum from different experiments. In this comparison, the change of the HHG spectra is due to the change of MWP which depends on the experimental setup. The averaged PR transition dipoles of HOMO and HOMO-2 are the same in the three calculations.

\section{F. The dependence of the HHG minimum on the pump-probe angle}

In Wörner et al. [49], it was found that the minimum in the HHG spectra of aligned $\mathrm{CO}_{2}$ moved to higher photon energy with increasing pump-probe angle $\alpha$, i.e., the 

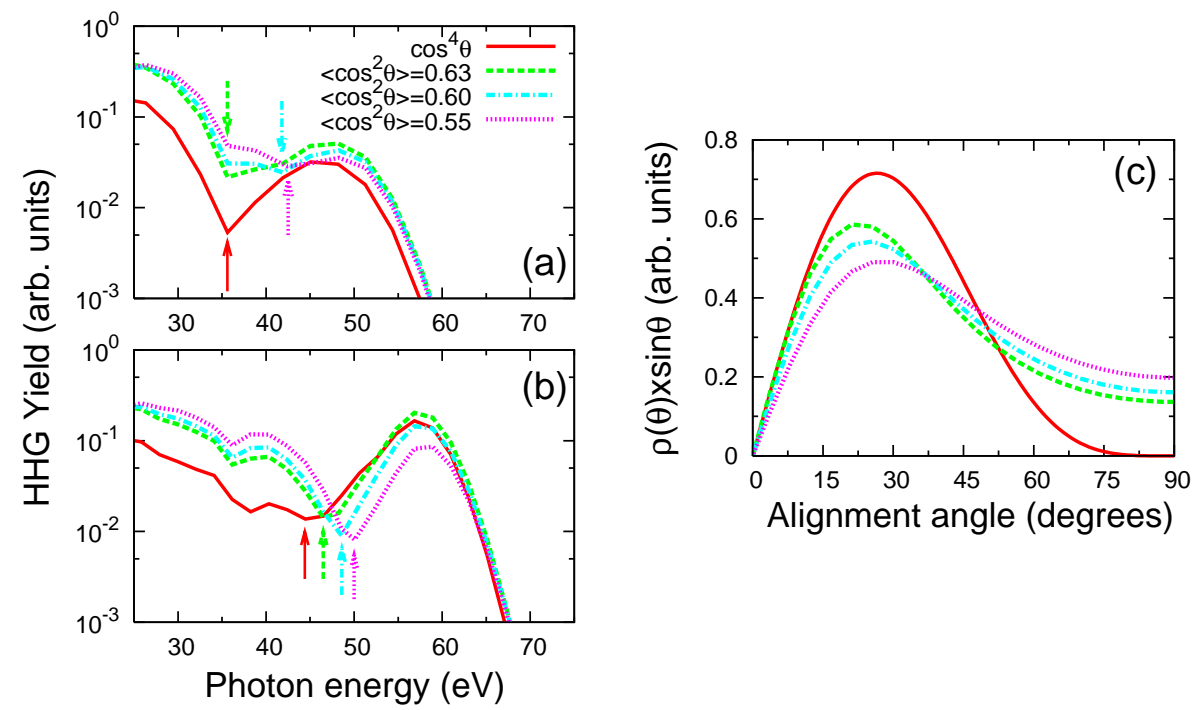

FIG. 4: (Color online) Dependence of macroscopic HHG spectra (envelope only) with degrees of molecular alignment distributions for (a) an 800-nm laser with intensity of $1.8 \times 10^{14} \mathrm{~W} / \mathrm{cm}^{2}$, and (b) a 1200 -nm laser with intensity of $1.0 \times 10^{14} \mathrm{~W} / \mathrm{cm}^{2}$. The weighted angular distributions of the molecules are shown in (c). Arrows indicate the positions of minima. Pump-probe angle $\alpha=0^{\circ}$.
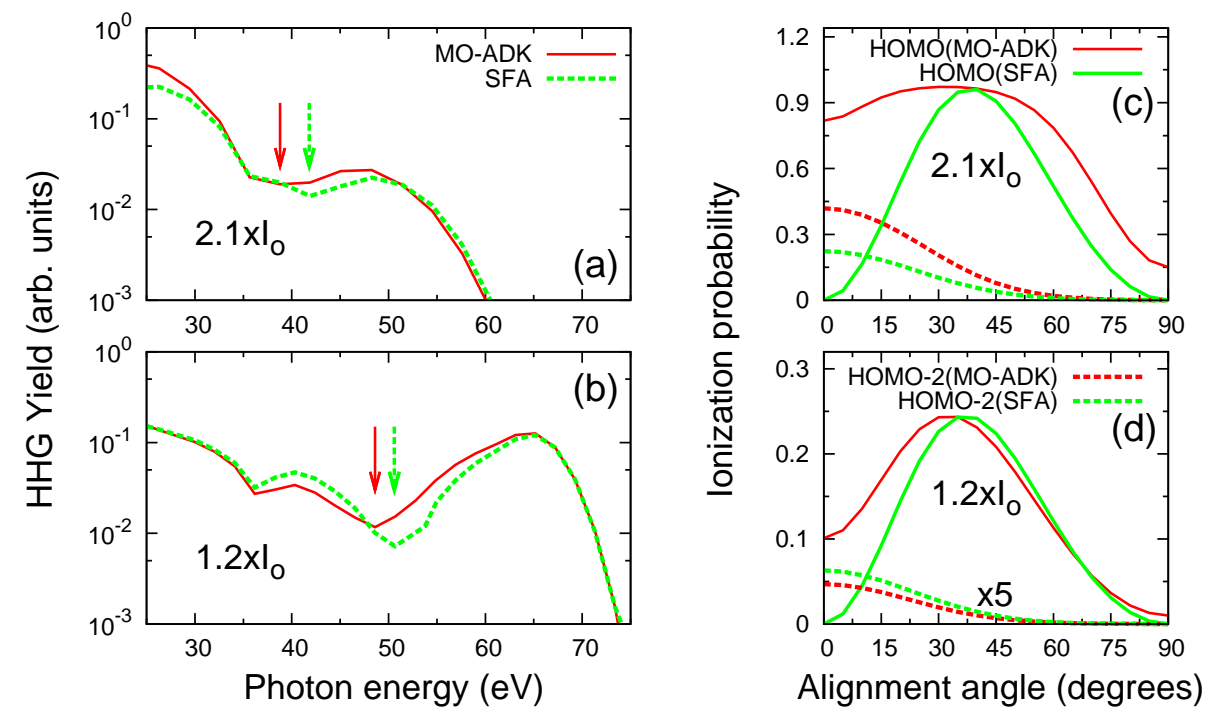

FIG. 5: (Color online) Dependence of macroscopic HHG spectra (envelope only) on the ionization probabilities calculated from MO-ADK or SFA in (a) an 800-nm laser, and (b) a 1200-nm laser. Laser intensities are indicated where $\mathrm{I}_{\circ}=10^{14} \mathrm{~W} / \mathrm{cm}^{2}$. Arrows indicate the positions of minima. Degree of alignment: $\left\langle\cos ^{2} \theta\right\rangle=0.60$. Pump-probe angle $\alpha=0^{\circ}$. (c) and (d) Alignmentdependent ionization probabilities of HOMO and HOMO-2 calculated using MO-ADK and SFA. Laser parameters are the same as (a) and (b). Ionization probabilities of HOMO-2 in (d) are multiplied by 5 .

angle between aligning pump beam and the HHG generating probe beam polarizations. This phenomenon has also been observed in other measurements [21, 69]. In contrast, the minimum in the HHG spectra of aligned $\mathrm{N}_{2}$ has been found to stay at the same position for all pump-probe angles [14, 69].

We show the calculated HHG spectra at four pumpprobe angles in an 800-nm laser in Fig. 7(a), and in a 1200-nm laser in Figs. 7(b) and 7(c). Laser parameters and experimental arrangements are the same as in Fig. 1 and the degree of alignment is $\left\langle\cos ^{2} \theta\right\rangle=0.60$. The HHG spectra for $\alpha=0^{\circ}$ have been shown in Fig. 3. These fig- ures show that the minimum in the HHG spectra moves to higher photon energies with increasing $\alpha$, and the minimum disappears at large angles. At larger pumpprobe angles, contributions from large alignment angles increase. Since HOMO dominates over HOMO-2 at large angles in both the ionization rates [see Figs. 5(c) and [5(d)] and the PR transition dipoles [see Figs. 7(d)-7(f)], thus HHG at large pump-probe angles has essentially no contributions from HOMO-2. Also note that at small $\alpha$, the total HHG yield is much smaller 34]. In fact, the total HHG spectra for randomly distributed $\mathrm{CO}_{2}$ have little contributions from molecules that are aligned nearly par- 


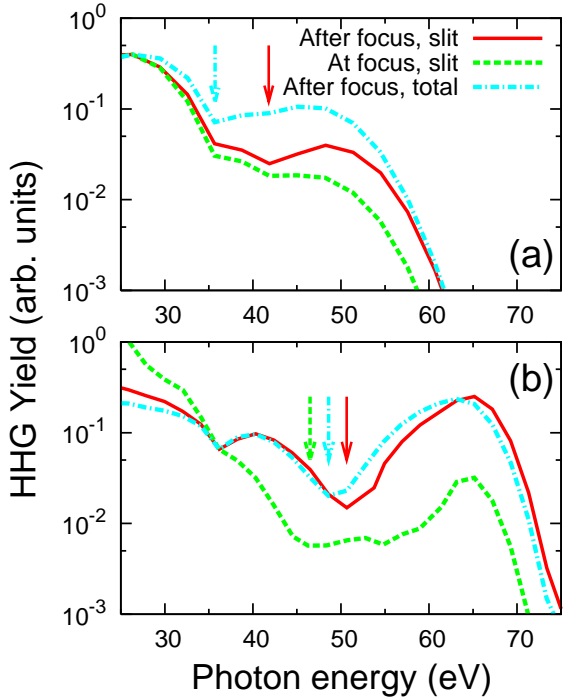

FIG. 6: (Color online) Dependence of macroscopic HHG spectra (envelope only) on experimental arrangements (a) for an 800 -nm laser with intensity of $2.1 \times 10^{14} \mathrm{~W} / \mathrm{cm}^{2}$, and (b) for a $1200-\mathrm{nm}$ laser with intensity of $1.2 \times 10^{14} \mathrm{~W} / \mathrm{cm}^{2}$. The arrangements are: (1) gas jet after focus and slit is used (solid lines); (2) gas jet at the focus and slit is used (dashed lines); and (3) gas jet is after the focus but without the slit (dotdashed lines). Arrows indicate the positions of minima. Degree of alignment: $\left\langle\cos ^{2} \theta\right\rangle=0.60$. Pump-probe angle $\alpha=0^{\circ}$.

allel to the polarization axis of the laser. The HHG spectra of $\mathrm{CO}_{2}$ are complex only in the region where HHG yields are small. This is generally true - interpretation of small processes always requires careful and detailed theories.

\section{SUMMARY AND OUTLOOK}

In this paper we have analyzed the multiple orbital contribution to $\mathrm{HHG}$ in $\mathrm{CO}_{2}$ with the inclusion of macroscopic propagation effect. In the past few years, there have been many experimental and theoretical studies on the $\mathrm{HHG}$ of $\mathrm{CO}_{2}$ from many laboratories, using lasers with different wavelengths and intensities, for $\mathrm{CO}_{2}$ molecules that are randomly distributed or partially aligned. In particular, for $\mathrm{CO}_{2}$ molecules that are partially aligned along the polarization axis of the probe laser, many experiments have shown that the HHG spectra exhibit minima and the positions of the minima shift with laser intensities [21, 23, 49]. The shift of the minimum position with laser intensities has been attributed to the interference between the contributions to HHG from the HOMO-2 with the one from the HOMO, despite
HOMO-2 lying at $4.4 \mathrm{eV}$ deeper than the HOMO. Since HHG is a nonlinear process, these observations posed a great challenge to the theory, especially for the prediction of the position of the minimum and how it changes with the experimental conditions. Since all experimental HHG spectra include macroscopic propagation effect, comparison of theory with experiment is incomplete unless the theory also has included the propagation effect. Our analysis in this paper is based on the macroscopic propagation code extended for aligned molecules, and the recently developed quantitative rescattering theory. We find that although HHG spectra change significantly under different experimental parameters such as degree of alignment, focussing condition, and the use of a slit, the position of the minimum in the HHG spectra behaves in a similar trend as laser intensity and pump-probe angle vary. This trend has been found to be consistent with the recent experimental measurements from different groups.

We comment that the present theory and the earlier one by Smirnova et al. [21] both explain the intensity dependence of the change of HHG minima, but the details between the two theories are quite different. The alignment dependence of the ionization rates, the recombination dipole matrix elements and their phases entering the two theories are not the same, for both the HOMO and HOMO-2. As illustrated in this paper, these parameters can all affect the position of the predicted interference minimum. Furthermore, in Smirnova et al. 21] the interference is attributed to the importance of hole dynamics in the ion core. Our approach is formulated in the time-independent fashion, no hole dynamics is included. Since HHG spectra are taken without explicit observation of electron dynamics, the difference between the two models cannot be settled. Despite these differences, our understanding of the HHG spectra of $\mathrm{CO}_{2}$ has come a long way since 2005 [26]. With the possibility of including macroscopic propagation effect "routinely" in the HHG theory for molecular targets, further experimental studies should explore the effects of laser focusing condition and gas pressure, for lasers extending to longer wavelengths. Such studies would further our basic understanding of strong-field physics of molecules to the next level, and eliminate the need of introducing extraneous assumptions in the interpretation of experimental data.

\section{ACKNOWLEDGMENTS}

This work was supported in part by Chemical Sciences, Geosciences and Biosciences Division, Office of Basic Energy Sciences, Office of Science, U.S. Department of Energy.
[1] C. Winterfeldt, C. Spielmann, and G. Gerber, Rev. Mod. Phys. 80, 117 (2008).
[2] T. Brabec and F. Krausz, Rev. Mod. Phys. 72, 545 (2000). 

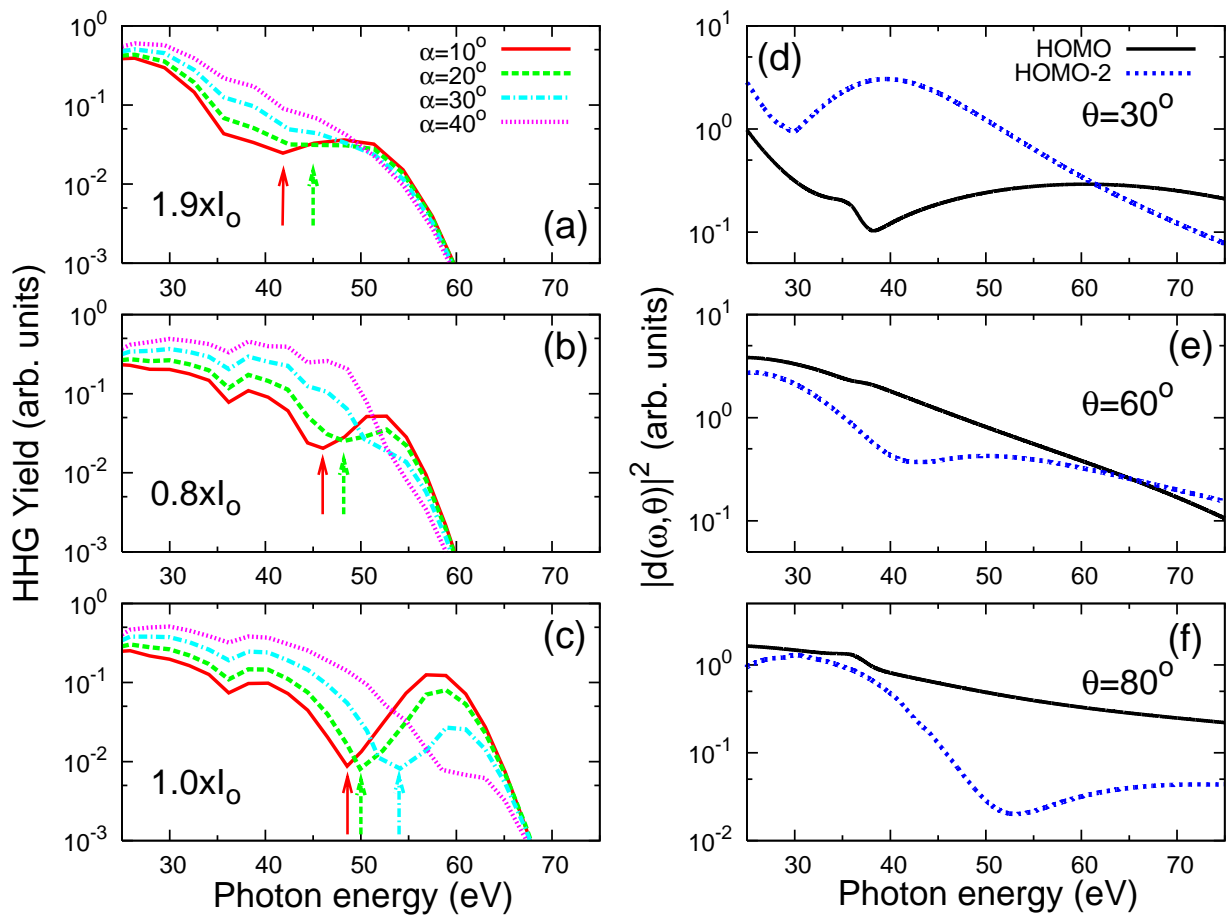

FIG. 7: (Color online) Dependence of macroscopic HHG spectra (envelope only) on pump-probe angles $\alpha=10^{\circ}, 20^{\circ}, 30^{\circ}$, and $40^{\circ}$ (a) for an 800-nm laser, (b) and (c) for a 1200-nm laser. Laser intensities are given in units of $\mathrm{I}_{\circ}=10^{14} \mathrm{~W} / \mathrm{cm}^{2}$. Arrows indicate the positions of minima. Degree of alignment: $\left\langle\cos ^{2} \theta\right\rangle=0.60$. (d)-(f) Photorecombination transition dipoles (parallel component, the square of magnitude) of HOMO and HOMO-2 in terms of photon energy at alignment angles $\theta=30^{\circ}, 60^{\circ}$, and $80^{\circ}$.

[3] P. Agostini and L. F. DiMauro, Rep. Prog. Phys. 67, 813 (2004).

[4] M. B. Gaarde, J. L. Tate, and K. J. Schafer, J. Phys. B 41, 132001 (2008).

[5] E. Priori, G. Cerullo, M. Nisoli, S. Stagira, S. De Silvestri, P. Villoresi, L. Poletto, P. Ceccherini, C. Altucci, R. Bruzzese, and C. de Lisio, Phys. Rev. A 61, 063801 (2000).

[6] V. Tosa, H. T. Kim, I. J. Kim, and C. H. Nam, Phys. Rev. A 71, 063807 (2005).

[7] M. Geissler, G. Tempea, A. Scrinzi, M. Schnürer, F. Krausz, and T. Brabec, Phys. Rev. Lett. 83, 2930 (1999).

[8] C. Jin, A. T. Le, and C. D. Lin, Phys. Rev. A 83, 023411 (2011).

[9] C. Altucci et al., Phys. Rev. A 73, 043411 (2006).

[10] M. C. H. Wong, J.-P. Brichta, and V. R. Bhardwaj, Phys. Rev. A 81, 061402 (2010).

[11] M. C. H. Wong, J.-P. Brichta, and V. R. Bhardwaj, Opt. Lett. 35, 1947 (2010).

[12] C. Trallero-Herrero et al., Chem. Phys. 366, 33 (2009).

[13] B. K. McFarland, J. P. Farrell, P. H. Bucksbaum, and M. Gühr, Science 322, 1232 (2008).

[14] S. Haessler et al., Nat. Phys. 6, 200 (2010).

[15] Y. Mairesse et al., Phys. Rev. Lett. 104, 213601 (2010).

[16] G. H. Lee, I. J. Kim, S. B. Park, T. K. Kim, Y. S. Lee, and C. H. Nam, J. Phys. B 43, 205602 (2010).

[17] W. Boutu et al., Nat. Phys. 4, 545 (2008).

[18] T. Kanai, E. J. Takahashi, Y. Nabekawa, and K. Midorikawa, Phys. Rev. A 77, 041402 (2008).

[19] N. Wagner et al., Phys. Rev. A 76, 061403 (2007).

[20] X. Zhou et al., Phys. Rev. Lett. 100, 073902 (2008).

[21] O. Smirnova, Y. Mairesse, S. Patchkovskii, N. Dudovich,
D. Villeneuve, P. Corkum, and M. Yu. Ivanov, Nature (London) 460, 972 (2009).

[22] C. Vozzi et al., Phys. Rev. Lett. 95, 153902 (2005).

[23] R. Torres et al., Phys. Rev. A 81, 051802 (2010).

[24] P. B. Corkum, Phys. Rev. Lett. 71, 1994 (1993).

[25] J. L. Krause, K. J. Schafer, and K. C. Kulander, Phys. Rev. Lett. 68, 3535 (1992).

[26] T. Kanai, S. Minemoto, and H. Sakai, Nature 435, 470 (2005).

[27] M. Lein, N. Hay, R. Velotta, J. P. Marangos, and P. L. Knight, Phys. Rev. Lett. 88, 183903 (2002).

[28] A. T. Le, X. M. Tong, and C. D. Lin, Phys. Rev. A 73, 041402 (2006).

[29] M. Lewenstein, Ph. Balcou, M. Yu. Ivanov, A. L'Huillier, and P. B. Corkum, Phys. Rev. A 49, 2117 (1994).

[30] X. X. Zhou, X. M. Tong, Z. X. Zhao, and C. D. Lin, Phys. Rev. A 71, 061801 (2005).

[31] X. X. Zhou, X. M. Tong, Z. X. Zhao, and C. D. Lin, Phys. Rev. A 72, 033412 (2005).

[32] C. D. Lin, A. T. Le, Z. Chen, T. Morishita, and R. R. Lucchese, J. Phys. B 43, 122001 (2010).

[33] T. Morishita, A. T. Le, Z. Chen, and C. D. Lin, Phys. Rev. Lett. 100, 013903 (2008).

[34] A. T. Le, R. R. Lucchese, S. Tonzani, T. Morishita, and C. D. Lin, Phys. Rev. A 80, 013401 (2009).

[35] A. T. Le, R. R. Lucchese, M. T. Lee, and C. D. Lin, Phys. Rev. Lett. 102, 203001 (2009).

[36] A. T. Le, R. R. Lucchese, and C. D. Lin, Phys. Rev. A 82, 023814 (2010).

[37] D. W. Turner, C. Baker, A. D. Baker, and C. R. Brundle, Molecular Photoelectron spectroscopy: a handbook of $\mathrm{He}$ 584 A spectra (Wiley, London, 1970). 
[38] C. D. Lin, X. M. Tong, and Z. X. Zhao, J. Mod. Phys. 53, 21 (2006).

[39] X. M. Tong, Z. X. Zhao, and C. D. Lin, Phys. Rev. A 66, 033402 (2002).

[40] S. F. Zhao, C. Jin, A. T. Le, T. F. Jiang, and C. D. Lin, Phys. Rev. A 81, 033423 (2010).

[41] D. Pavičić, K. F. Lee, D. M. Rayner, P. B. Corkum, and D. M. Villeneuve, Phys. Rev. Lett. 98, 243001 (2007).

[42] V.-H. Le, N.-T. Nguyen, C. Jin, A.-T. Le, and C. D. Lin, J. Phys. B 41, 085603 (2008).

[43] M. Spanner and S. Patchkovskii, Phys. Rev. A 80, 063411 (2009).

[44] S. F. Zhao, C. Jin, A. T. Le, T. F. Jiang, and C. D. Lin, Phys. Rev. A 80, 051402 (2009).

[45] M. Abu-samha and L. B. Madsen, Phys. Rev. A 80, 023401 (2009).

[46] S. K. Son and Shih-I. Chu, Phys. Rev. A 80, 011403 (2009).

[47] S. Petretti, Y. V. Vanne, A. Saenz, A. Castro, and P. Decleva, Phys. Rev. Lett. 104, 223001 (2010).

[48] A. T. Le, R. R. Lucchese, and C. D. Lin, J. Phys. B 42, 211001 (2009).

[49] H. J. Wörner, J. B. Bertrand, P. Hockett, P. B. Corkum, and D. M. Villeneuve, Phys. Rev. Lett. 104, 233904 (2010).

[50] M. B. Gaarde, M. Murakami, and R. Kienberger, Phys. Rev. A 74, 053401 (2006).

[51] M. B. Gaarde and K. J. Schafer, Phys. Rev. A 65, 031406 (2002).

[52] A. T. Le, R. D. Picca, P. D. Fainstein, D. A. Telnov, M. Lein, and C. D. Lin, J. Phys. B 41, 081002 (2008).

[53] S. F. Zhao, C. Jin, R. R. Lucchese, A. T. Le, and C. D.
Lin, Phys. Rev. A (to be published).

[54] C. Jin, A. T. Le, and C. D. Lin, Phys. Rev. A 79, 053413 (2009).

[55] C. Jin, H. J. Wörner, V. Tosa, A. T. Le, J. B. Bertrand, R. R. Lucchese, P. B. Corkum, D. M. Villeneuve, and C. D. Lin (unpublished); e-print arXiv:1012.1892,

[56] J. P. Farrell et al., Phys. Rev. A 83, 023420 (2011).

[57] J. Higuet et al. (unpublished); e-print arXiv:1012.2676.

[58] C. Jin, A. T. Le, S. F. Zhao, R. R. Lucchese, and C. D. Lin, Phys. Rev. A 81, 033421 (2010).

[59] M. Lein et al., J. Mod. Opt. 52, 465 (2005).

[60] A. E. Siegman, Lasers (University Science, Mill Valley, CA, 1986).

[61] A. L'Huillier, Ph. Balcou, S. Candel, K. J. Schafer, and K. C. Kulander, Phys. Rev. A 46, 2778 (1992).

[62] V. Tosa, K. T. Kim, and C. H. Nam, Phys. Rev. A 79, 043828 (2009).

[63] R. R. Lucchese and V. McKoy, Phys. Rev. A 26, 1406 (1982).

[64] R. R. Lucchese, G. Raseev, and V. McKoy, Phys. Rev. A 25, 2572 (1982).

[65] C. B. Madsen and L. B. Madsen, Phys. Rev. A 74, 023403 (2006).

[66] C. Figueira de Morisson Faria and B. B. Augstein, Phys. Rev. A 81, 043409 (2010).

[67] R. Torres et al., Opt. Express 18, 3174 (2010).

[68] O. Smirnova, S. Patchkovskii, Y. Mairesse, N. Dudovich, and M. Yu. Ivanov, Proc. Natl. Acad. Sci. U.S.A. 106, 16556 (2009).

[69] Y. Mairesse, J. Levesque, N. Dudovich, P. B. Corkum, and D. M. Villeneuve, J. Mod. Opt. 55, 2591 (2008). 